\title{
The Plexin-B1/Rac interaction inhibits PAK activation and enhances Sema4D ligand binding
}

\author{
Haris G. Vikis, ${ }^{1}$ Weiquan Li, ${ }^{1}$ and Kun-Liang Guan ${ }^{1,2,3}$ \\ ${ }^{1}$ Department of Biological Chemistry, University of Michigan Medical School, Ann Arbor, Michigan 48109, USA; \\ ${ }^{2}$ Institute of Gerontology, University of Michigan, Ann Arbor, Michigan 48109, USA
}

\begin{abstract}
The small GTPase Rac has been implicated in growth cone guidance mediated by semaphorins and their receptors. Here we demonstrate that plexin-B1, a receptor for Semaphorin4D (Sema4D), and p21-activated kinase (PAK) can compete for the interaction with active Rac and plexin-B1 can inhibit Rac-induced PAK activation. We have also demonstrated that expression of active Rac enhances the ability of plexin-B1 to interact with Sema4D. Active Rac stimulates the localization of plexin-B1 to the cell surface. The enhancement in Sema4D binding depends on the ability of Rac to bind plexin-B1. These observations support a model where signaling between Rac and plexin-B1 is bidirectional; Rac modulates plexin-B1 activity and plexin-B1 modulates Rac function.
\end{abstract}

[Key Words: Plexin-B1; Rac; Semaphorin4D; PAK]

Received November 30, 2001; revised version accepted February 13, 2002.

During embryonic development emerging neurons are guided to their targets by extracellular cues in the environment (Tessier-Lavigne and Goodman 1996). Semaphorins have been identified as one of these chemo-repulsive cues (Puschel 1996). Many semaphorins have been shown to cause growth cone collapse and repulse neurons away from a semaphorin gradient (Luo et al. 1993; Messersmith et al. 1995; Puschel et al. 1995; Culotti and Kolodkin 1996). Recently it was shown that transmembrane neuropilins in complex with transmembrane plexins form functional receptors for the soluble class of semaphorins while another subset of plexins acting alone function as receptors for the membrane-bound class of semaphorins (Takahashi et al. 1999; Tamagnone et al. 1999; Rohm et al. 2000a). Signaling downstream of plexins is mediated by their intracellular domain as deletion of this domain abolishes semaphorin-induced growth cone collapse/repulsion (Takahashi et al. 1999; Tamagnone et al. 1999).

Growth cone collapse/repulsion is the result of dramatic actin cytoskeletal reorganization involving actin disassembly and withdrawal of lamellipodia and filopodia (Fan et al. 1993; O’Connor and Bentley 1993; Bentley and O'Connor 1994; Jin and Strittmatter 1997; Kuhn et al. 1999). This has implicated the Rho family of small GTPases (Rho, Rac, and cdc42) in signaling downstream

${ }^{3}$ Corresponding author.

E-MAIL kunliang@umich.edu; FAX (734) 763-4581.

Article and publication are at http://www.genesdev.org/cgi/doi/10.1101/ gad.966402. of plexins. Insight into the role of Rho GTPases in semaphorin signaling came from initial experiments where trituration of dorsal root ganglia (DRG) with active Rac caused growth cone collapse, whereas dominant negative Rac was able to block Sema3A-induced growth cone collapse (Jin and Strittmatter 1997). However, these results suggest that Rac activity is required in growth cone collapse and conflict with the view that Rac is inactivated during cytoskeletal disassembly. Hence the precise role of Rac in semaphorin-mediated growth cone collapse is not fully understood.

We and others have previously identified and characterized the interaction between active Rac and the intracellular domain of plexin-B1, a receptor for Sema4D (Rohm et al. 2000b; Vikis et al. 2000; Driessens et al. 2001). At present, the functional consequence of the plexin-B1/Rac interaction is not understood. In this report, we have shown that plexin-B1 can compete with p21-activated kinase (PAK) for binding to active Rac. This competition results in the ability of plexin-B1 to inhibit Rac-induced PAK activation. Furthermore, we observed that active Rac enhances the binding of Sema4D to plexin-B1. This is mediated by direct binding of Rac to plexin. We have also shown that endogenous $\mathrm{Rac}$ is required for basal levels of plexin-B1 ligand binding. The enhancement of ligand binding caused by active $\mathrm{Rac}$ is a result of an increase in the amount of receptor at the cell surface. Our observations suggest that the plexin-B1/Rac interaction has two functions: to inhibit Rac stimulation of PAK by sequestration of Rac, and to enhance the interaction with Sema4D ligand. 
Results

\section{Plexin-B1 and PAK compete for Rac binding}

To study the functional significance of the plexin-B1 interaction with Rac we postulated that plexin-B1 may compete with known Rac effectors for Rac binding. PAK is a known Rac effector whose kinase activity is enhanced by the binding of Rac to its N-terminal Rac-binding domain (RBD) (Knaus and Bokoch 1998). We found that a glutathione- $S$-transferase (GST)-fusion of the $\mathrm{N}$ terminal RBD of PAK (residues 71-151 of mouse PAK-A) was able to effectively inhibit the interaction of plexinB1 and the activated Rac mutant, RacL61, in vitro, whereas GST alone was not able to (Fig. 1A). Mutations of residue 37 , but not 40 , in the Rac effector domain eliminated plexin-B1 binding (Fig. 1B, upper panel; Vikis et al. 2000; Driessens et al. 2001) whereas mutation of residue 40 abolishes the interaction with PAK (Lamarche et al. 1996). Consistent with these data, we found that RacL61C40 interacts with plexin-B1 and this interaction cannot be competed for by PAK-RBD (Fig. 1B, lower panel).
Plexin-B1 inhibits Rac-induced PAK activation

Coexpression of active Rac or Cdc42 and PAK in mammalian cells results in the enhancement of PAK kinase activity (Lim et al. 1996). Binding of Cdc42-GTP to the N-terminal domain, induces conformational changes that result in kinase activation (Buchwald et al. 2001). We postulated that expression of the cytoplasmic domain of plexin-B1 (plexin-B1 ${ }_{\text {cyto }}$ amino acids 1512-2135) would inhibit Rac-induced PAK activation by competition and sequestration of active Rac. Expression of RacL61 significantly enhanced PAK activity in mammalian HEK293 cells as measured by in vitro kinase assay and $\alpha$-phospho-PAK antibody that recognizes phosphorylated active PAK (Fig. 1C). Coexpression of plexin-B1 $1_{\text {cyto }}$ effectively inhibited RacL61-induced PAK activity (Fig. 1C). These data suggest that plexin-B1 is able to sequester active Rac from interacting with PAK and effectively inhibit Rac-induced PAK activity. While this manuscript was in preparation, $\mathrm{Hu}$ et al. (2001) reported that Drosophila Plexin B (PlexB) competes with PAK for Rac binding and that the inhibition of Rac activity is important for PlexB mediated axon guidance in Drosophila (Hu

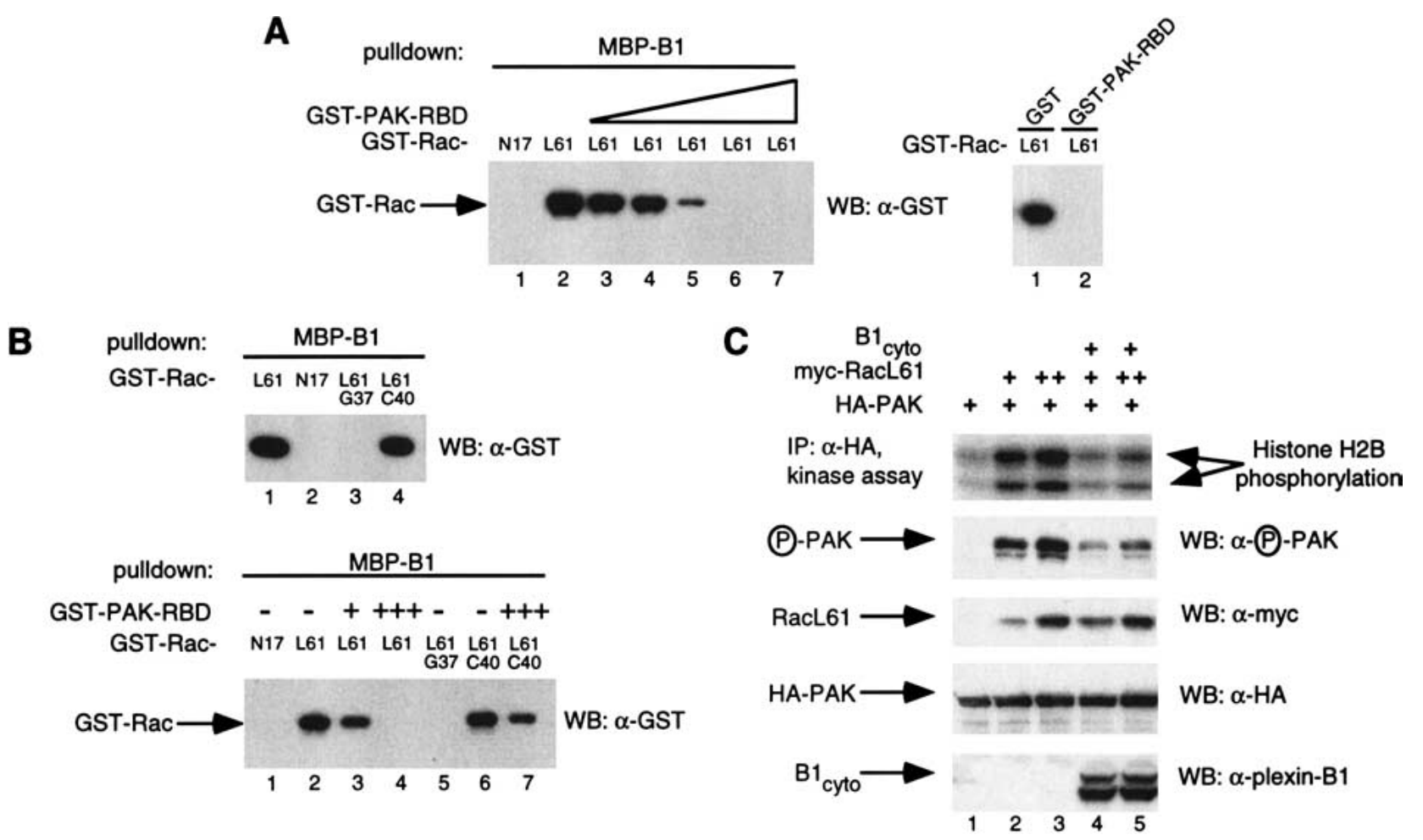

Figure 1. (A) The Rac-binding domain (RBD) of p21-activated kinase (PAK) competes with plexin-B1 for RacL61. In vitro binding was performed using purified glutathione $S$-transferase (GST)-RacN17 (5 $\mu$ g, lane 1) or GST-RacL61 (lane 2) and incubated with MBPplexin-B1 $(10 \mu \mathrm{g})$ prebound to amylose resin in the absence or presence of increasing amounts of GST-PAK-RBD (lane 3, $0.5 \mu \mathrm{g} ;$ lane 4, $1 \mu \mathrm{g}$; lane 5, $5 \mu \mathrm{g}$; lane 6, $15 \mu \mathrm{g}$; lane 7, $25 \mu \mathrm{g}$, left panel). Similarly, $15 \mu \mathrm{g}$ of GST or GST-PAK-RBD were used (right panel). Associated GST-Rac was isolated and subject to Western blot with $\alpha$-GST antibody (Zymed). (B) The RBD of PAK can compete with plexin-B1 for RacL61G37, but not RacL61C40. MBP-B1 (10 $\mu \mathrm{g})$ prebound to amylose resin was subjected to in vitro binding with GST-RacL61/N17/L61G37/L61C40 (5 g) (upper panel). Bound GST-Rac was determined by $\alpha$-GST Western blot. GST-RacN17/L61 $(5 \mu \mathrm{g})$ was incubated with MBP-plexin-B1 $(10 \mu \mathrm{g})$ prebound to amylose resin in the presence or absence of GST-PAK-RBD (lane 3, 1 $\mu \mathrm{g}$; lanes 4 and 7, $15 \mu \mathrm{g}$ ) (lower panel). (C) Plexin B1 inhibits RacL61-induced PAK activation. HEK 293 cells were transfected with 200 ng of HA-PAK, $500 \mathrm{ng}$ plexin-B1 $1_{\text {cyto }}$ and $25 \mathrm{ng}$ or $50 \mathrm{ng}$ myc-RacL61. HA-PAK was immunoprecipitated with $\alpha$-HA (BAbCO). Kinase activity was determined by in vitro kinase assay using histone $\mathrm{H} 2 \mathrm{~B}$ as a substrate or by $\alpha$-phospho PAK antibody. 
Vikis et al.

A
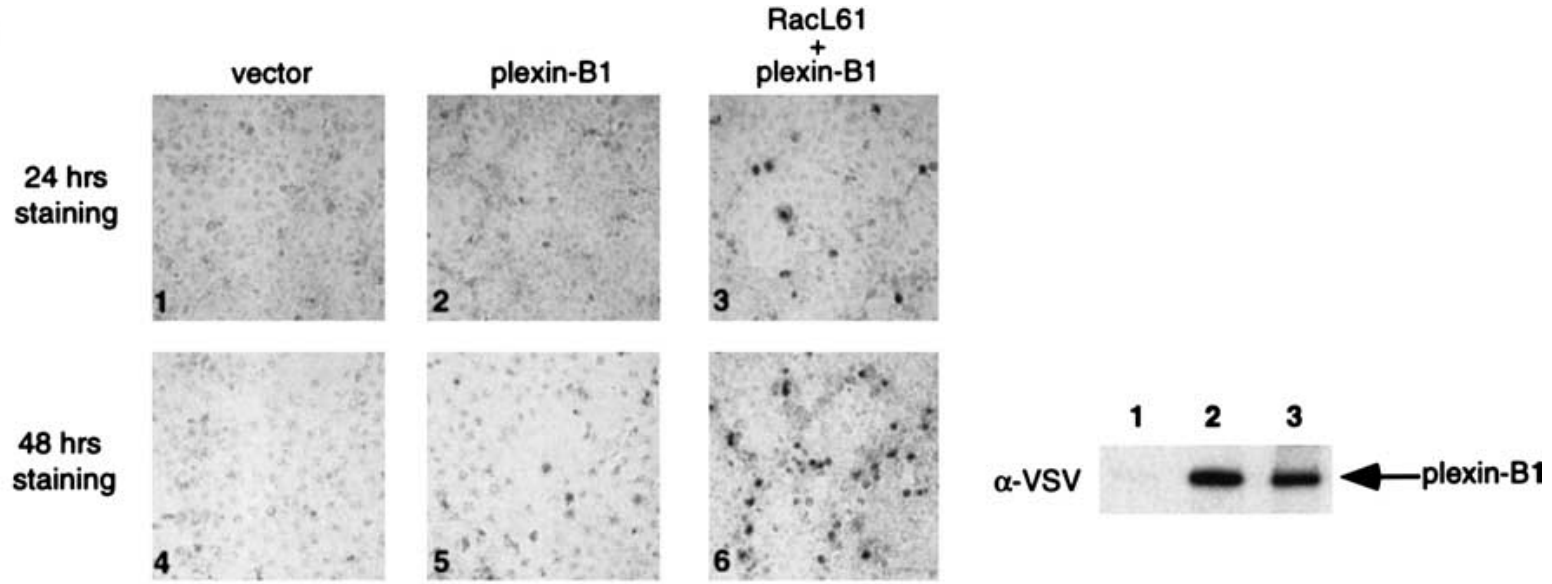

$72 \mathrm{hrs}$ staining
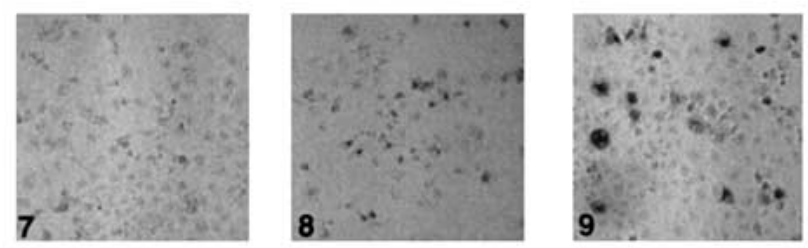

B
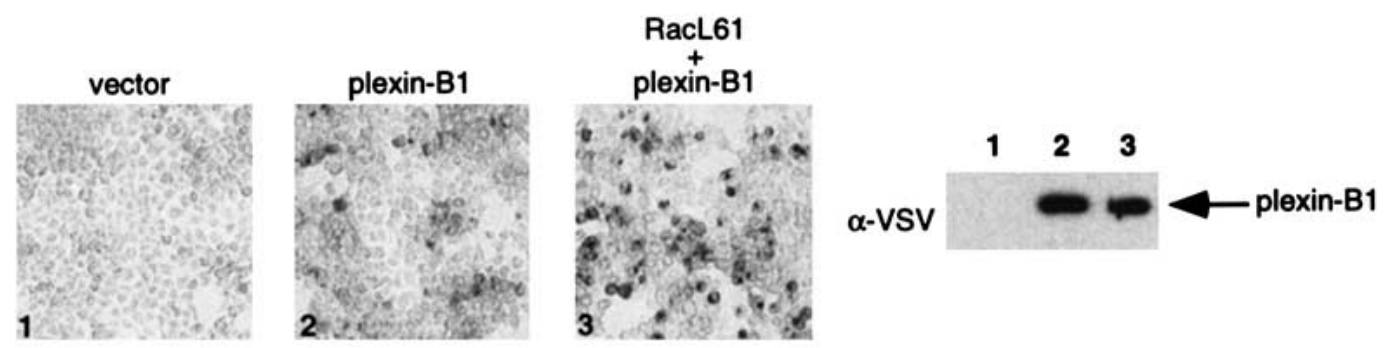

C
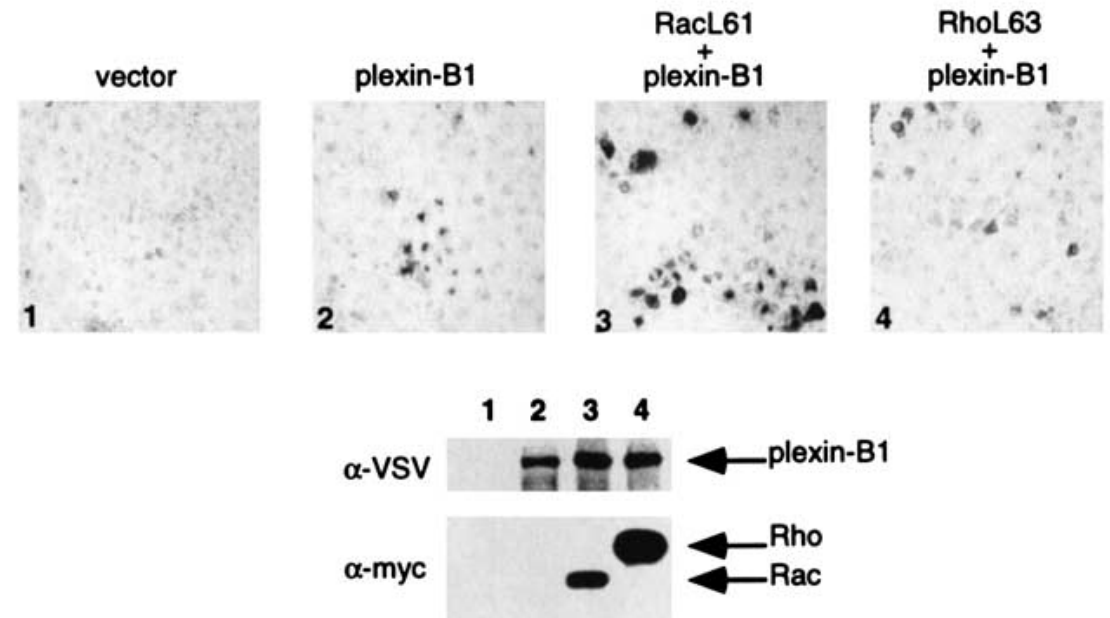

D
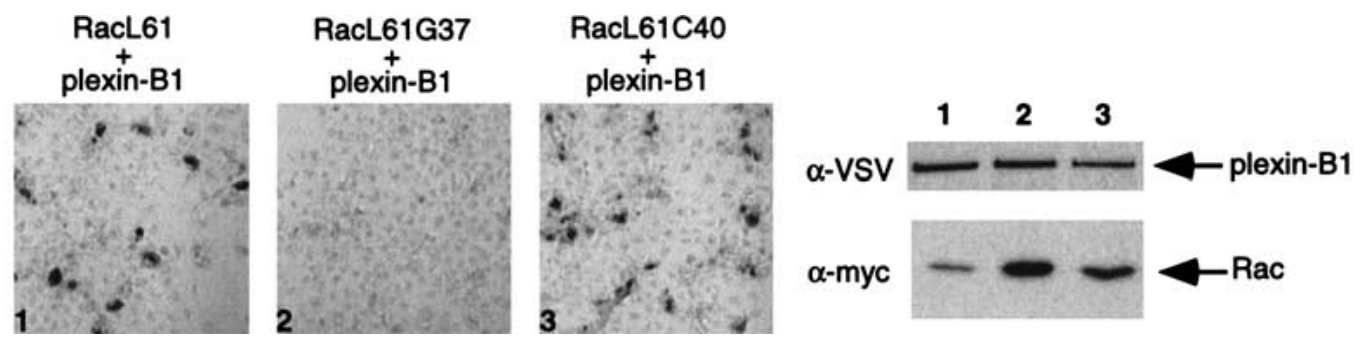

(Figure 2 continued on facing page) 

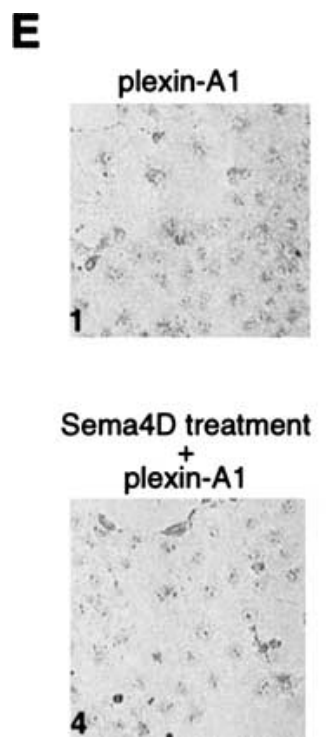

NP-1

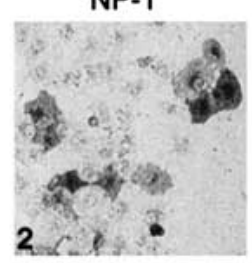

RacN17

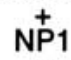

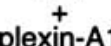

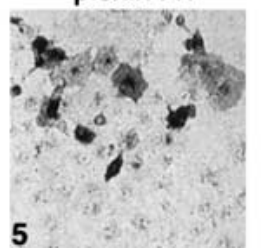

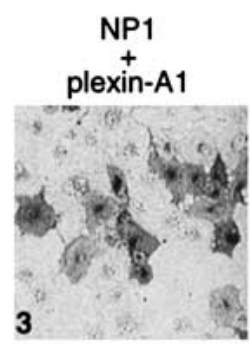

RacL61

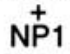

$+$
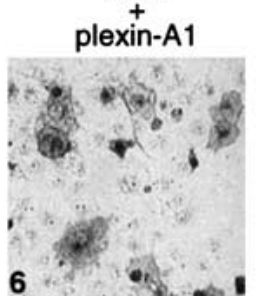

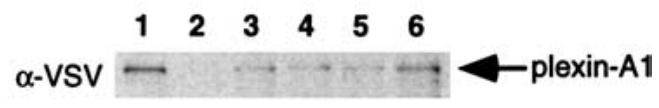

Figure 2. (A) Expression of RacL61 enhances the binding of Sema4D to plexin-B1 in COS-7 cells. COS-7 cells were transfected with expression vectors for VSV-plexin-B1, mycRacL61, or pcDNA3. Binding of AP-Sema4D was performed. Pictures were taken at 100x magnification and show staining over the course of $24 \mathrm{~h}$ (panels 1-3), $48 \mathrm{~h}$ (panels 4-6), and $72 \mathrm{~h}$ (panels 7-9). The expression level of plexin-B1 from a duplicate plate was determined by $\alpha$-VSV Western blot. Lanes 1-3 correspond to panels 1-3, respectively. Differences in background color are attributable to differences in shutter speed of the digital camera. $(B)$ Expression of RacL61 enhances the binding of Sema4D to plexin-B1 in HEK293 cells. Cells were transfected as in $A$, however staining is shown after 5 h. $(C)$ RhoL63 does not enhance the binding of Sema4D to plexin-B1. COS-7 cells were transfected with the indicated expression vectors and subject to the same staining procedures as in $A$. (D) Mutations in RacL61 ablate the ability to stimulate binding of plexin-B1 to Sema4D. Transfections and staining were carried out as in $A$. (E) Rac does not affect the interaction of Sema3A with NP-1/Plexin-A1. COS-7 cells were transfected with the indicated expression vectors. Cells were treated with Sema3A for $1 \mathrm{~h}$ prior to fixation and staining as described in A. Sema4D ligand was used in panel 4 and $400 \times$ magnification was used to take the photos.

et al. 2001). Our data confirm and extend these observations in the mammalian system.

\section{Active Rac enhances the interaction of plexin-B1 with Sema4D}

Previous studies have established that Sema4D is the ligand for the plexin-B1 receptor (Tamagnone et al. 1999). We also observed that an alkaline phosphatase (AP)-Sema4D fusion protein bound plexin-B1 expressed in COS-7 cells (Fig. 2A). We found that coexpression of RacL61 dramatically enhanced Sema4D binding (Fig. 2A). The enhanced binding caused by RacL61 was also observed in a number of cell lines including COS-1 (data not shown) and HEK293 (Fig. 2B). We also tested the effect of RhoA on plexin-B1/Sema4D binding. Interestingly, active RhoA (RhoAL63) was not able to enhance plexin-B1/Sema4D binding (Fig. 2C).

We have shown previously that the effector domain mutant, RacL61C40 is able to interact with plexin-B1, but that RacL61G37 does not (Fig. 1B). Consistent with their ability to bind plexin-B1, RacL61C40 enhanced the receptor/ligand interaction and RacL61G37 did not (Fig. 2D). This suggests that the ability of Rac to enhance plexin-B1/Sema4D binding is associated with its ability to bind the receptor.

Rho family GTPases have been implicated to function downstream of NP-1/plexin-A1 receptor complex (Liu and Strittmatter 2001). Although Rac does not interact directly with the plexin-A family of receptors (Rohm et al. 2000b; Vikis et al. 2000; Driessens et al. 2001), we tested whether expression of active Rac in COS-7 cells could affect the interaction between Sema3A and NP-1/ plexin-A1. We found that expression of either active or dominant negative Rac did not affect the interaction of Sema3A with NP-1/plexin-A1, consistent with the observation that plexin-A1 does not interact with Rac (Fig. 2E). These observations suggest that the direct interaction of plexin-B1 and RacL61 is responsible for the enhanced binding of Sema4D with plexin-B1.

\section{Rac enhances plexin-B1 affinity and localization to the cell membrane}

The ability of active Rac to enhance the plexin-B1/ Sema4D interaction can occur via two mechanisms: (1) increasing the receptor's affinity for ligand, and/or (2) increasing the number of receptors at the cell membrane. To test these hypotheses, COS-7 cells were transfected with plexin-B1 in the presence and absence of RacL61 and incubated with varying concentrations of APSema4D ligand. We observed that cells coexpressing RacL61 bound more Sema4D as measured by an increase in the amount of alkaline phosphatase activity (Fig. 3A) that was also observed in 293 cells (data not shown). This suggested that RacL61 may enhance the amount of 
plexin-B1 receptor at the cell surface. Scatchard analysis of the binding revealed that cells expressing both plexinB1 and RacL61 also indicated a trend towards enhanced affinity for Sema4D compared to cells expressing plexinB1 alone (Fig. 3A; data not shown). These binding data suggest that expression of active Rac results predominantly in enhanced plexin-B1 localization to the cell surface with a minor enhancement of receptor affinity.

If RacL61 enhances plexin-B1 localization to the cell surface we postulated that these receptors would be more susceptible to cleavage of the extracellular domain by proteinase $\mathrm{K}$. Treatment with proteinase $\mathrm{K}$ revealed that in the presence of RacL61, plexin-B1 is more sensitive to proteinase $\mathrm{K}$ cleavage than cells in the absence of RacL61 (Fig. 3B). We observed that the full-length receptor is cleaved into a smaller form whose appearance is detected by plexin-B1 antibody that was raised against the plexin-B1 cytosolic domain. Furthermore, expression of RacL61 enhanced cell surface biotinylation of the plexin-B1 receptor (Fig. 4B). These data are consistent with the model that RacL61 binds plexin-B1 and enhances plexin-B1 expression at the cell surface.

The Rac-binding deficient mutant plexin-B1-GGA is defective in Sema4D binding and cannot be stimulated by RacL61

We have shown previously that mutation of residues L1849,V1850, and P1851 (plexin-B1-GGA), which are located in the plexin-B1 cdc42/Rac interactive binding motif (CRIB, residues 1843-1889), ablate the interaction with active Rac (Vikis et al. 2000). It is possible that the inability of RacL61G37 to stimulate the plexin-B1/ Sema4D interaction is not attributable to loss of binding, but is caused by a defect in signaling to other downstream events that modulate plexin-B1/Sema4D binding. To further test the role of Rac binding in modulation of the plexin-B1/Sema4D interaction, we examined the effect of RacL61 on the binding of Sema4D to plexin-B1GGA. Our results show that the binding of Sema4D to plexin-B1-GGA was not stimulated by RacL61 (Fig. 4A). However, mutation of R1677 and R1678 (residues which are conserved in all plexin family members and correspond to the catalytic arginine found in RasGAP like domains) to methionine (plexin-B1-MM) still permitted RacL61 to stimulate the interaction with ligand. These observations further support that a direct interaction is required for Rac to stimulate plexin-B1/Sema4D binding.

We also observed that plexin-B1-GGA transfected cells displayed much lower basal Sema4D binding compared to wild-type plexin-B1, although the plexin-B1GGA mutant was expressed at similar levels (Fig. 4A). This suggests that the interaction of plexin-B1 with endogenous Rac is important for the proper function/localization of the receptor. To further determine the cell surface localization of plexin-B1, biotinylation of cell surface proteins was performed with membrane impermeable sulfo-NHS-LC-biotin. We observed that plexinB1-GGA was more weakly cell surface biotinylated than wild-type plexin-B1 which suggests that less of it is localized at the cell surface (Fig. 4B). Consistent with previous results (Figs. 2,3), RacL61 enhanced the biotinylation of plexin-B1 (Fig. 4B). To further test the role of endogenous Rac on plexin-B1 function, we blocked endogenous Rac activity by coexpression with dominant negative Rac (RacN17). Expression of RacN17 significantly inhibited the binding of plexin-B1 to Sema4D (Fig. 4C). Together our data demonstrate that plexin-B1/ Sema4D binding is regulated by endogenous Rac-GTP.

\section{PAK inhibits Rac stimulated plexin-B1 binding to Sema4D}

We have shown that expression of the intracellular domain of plexin-B1 interferes with PAK activation by sequestration of active Rac (Fig. 1) and expression of active Rac can promote the binding of plexin-B1 with Sema4D (Fig. 2). Therefore, we tested whether expression of the $\mathrm{N}$-terminal domain of PAK (residues 1-251) could interfere with the RacL61-induced plexin-B1/Sema4D interaction. We observed that expression of the $\mathrm{N}$-terminal domain of PAK (amino acids 1-251), which encompasses the RBD was able to inhibit the enhancement of Sema4D binding caused by active Rac (Fig. 4D). These data further support that the binding of Rac to PAK or plexin-B1 is mutually exclusive in vivo.

\section{Discussion}

We have reported previously that Rac-GTP interacts with the intracellular domain of plexin-B1 (Vikis et al. 2000). The intracellular domain of plexins share significant sequence homology with the RasGAP family of GTPase activating proteins (GAPs) (Rohm et al. 2000b). However, we have been unable to demonstrate that plexin-B1 directly affects the nucleotide status of Rac (data not shown). In this study we observed that plexinB1 competes with PAK for binding to active Rac in vitro. In vivo, we observed that overexpression of the cytoplasmic domain of plexin-B1 inhibited the activation of PAK by Rac. This suggests that one function of plexin-B1 is to inhibit PAK activation through sequestration of Rac. Interestingly, PAK has been implicated in mediating various cytoskeletal changes in both neuronal and nonneuronal cells. PAK protein has been shown to be involved in regulation of filopodia and membrane ruffles (Sells et al. 1997; Frost et al. 1998) and localize to membrane ruffles and edges of lamellipodia in fibroblast cells (Dharmawardhane et al. 1997). In Drosophila, PAK plays a critical role in photoreceptor axon guidance (Hing et al. 1999; Newsome et al. 2000). Hence, down-regulation of PAK by Sema4D/Plexin-B1 correlates with processes found in collapsing/turning growth cones, i.e., the rapid disassembly of cytoskeletal structures. Recently, Hu et al. (2001) reported genetic evidence that Drosophila plexB mediates axon guidance by inhibiting Rac activity and simultaneously enhancing Rho activity. They observed that overexpression of PAK antagonized a plexB gain of function phenotype, a phenotype that is aug- 
A

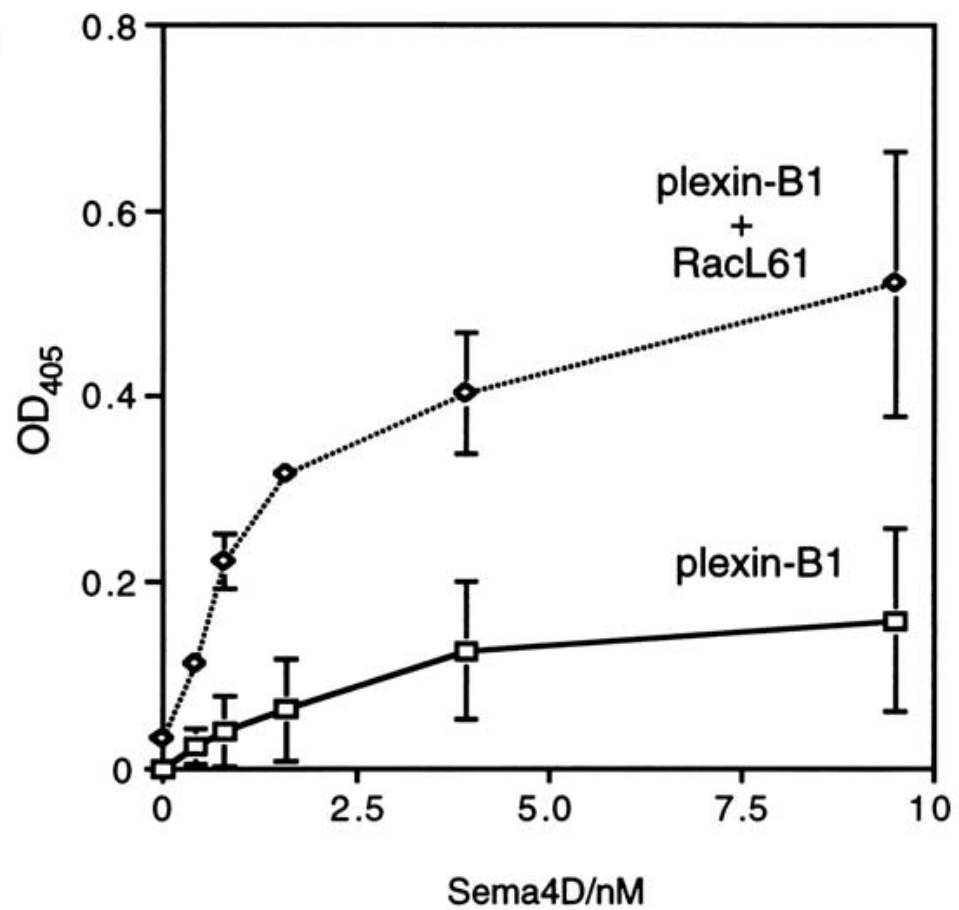

B

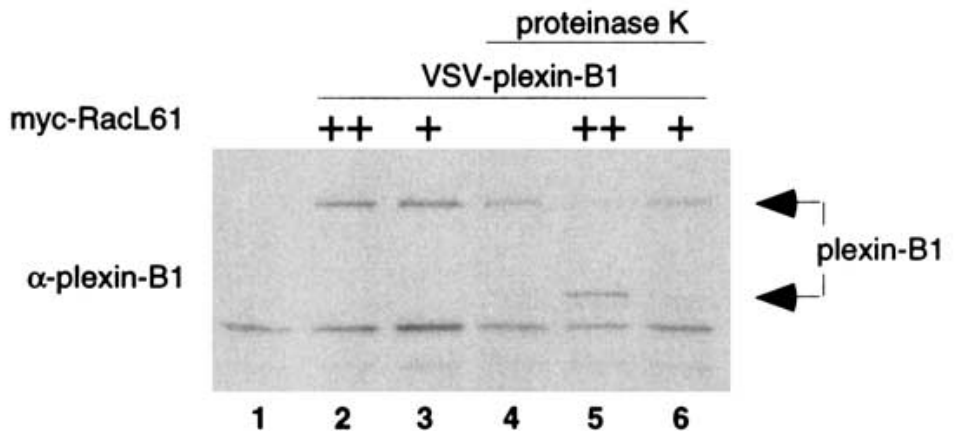

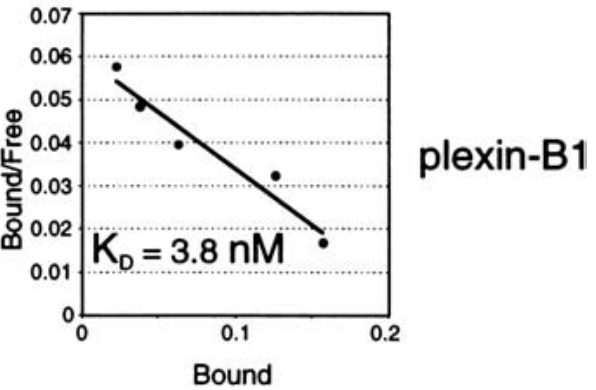

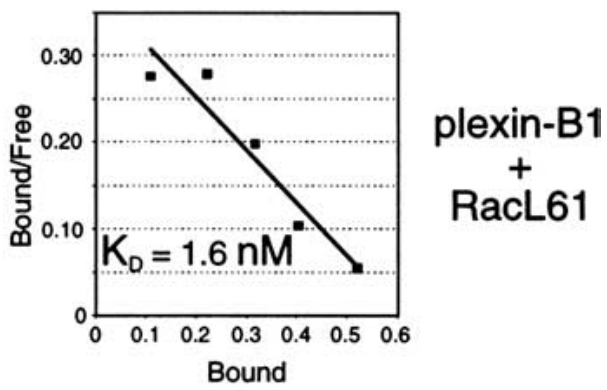

Figure 3. (A) RacL61 enhances the affinity and number of plexin-B1 receptors at the cell surface. COS-7cells were transfected as described in Materials and Methods. AP-Sema4D binding was assayed as described in Materials and Methods. Background activity of vector-transfected cells was subtracted. Scatchard plots are shown and linear regression analysis was used to determine the line of best fit. The data shown is an average of three replicates with error bars indicating the calculated standard deviation. (B) RacL61 recruits plexin-B1 to the cell surface. HEK293 cells were transfected with VSVplexin-B1 (1 $\mu \mathrm{g}$ ) and myc-RacL61 (25 ng or $100 \mathrm{ng}$ ) in the combinations indicated. Cells were treated with $0.2 \mathrm{mg} / \mathrm{mL}$ proteinase $\mathrm{K}$ and lysates were analyzed by Western blot with anti-plexin-B1 antibody. mented by active Rac. Our biochemical analysis using the mammalian proteins is consistent with their genetic observations.

We have demonstrated previously that Sema4D enhances the interaction between plexin-B1 and active Rac (Vikis et al. 2000). This observation allows us to propose a model by which Sema4D binds the plexin-B1 receptor and stimulates the recruitment of Rac-GTP (Fig. 5A). Sequestration of Rac results in the inactivation of PAK and growth cone collapse/turning. This model is supported by genetic data by Hu et al. (2001). However, this model conflicts with studies on the role of Rac downstream of the plexin-A1 receptor where dominant negative Rac inhibits collapse in response to Sema3A, which suggests that Rac activation is required for Sema3A-mediated growth cone collapse (Jin and Strittmatter 1997). Perhaps plexin-A and -B signal via different mechanisms as plexin-A does not interact with active Rac. However, Hu et al. (2001) have shown that in Drosophila Rac functions downstream of plexA even though it does not in- teract with plexA. It is possible that a yet unidentified protein couples plexin-A with Rac.

The regulation of Rho family GTPases in response to various ligands has become a central theme in growth cone repulsion/attraction studies. Recently, Shamah et al. (2001) have shown how the EphrinA ligand and EphA receptor mediate short-range repulsive cues through small GTPases. Similarly, the repulsive signals mediated by the Slit ligand and $\mathrm{ROBO}$ receptor were found to down-regulate the small GTPase, Cdc42 (Wong et al. 2001). It is now becoming clear that down-regulation of either Rac or Cdc42 are common features resulting in growth cone repulsion/collapse.

Our results indicate that another consequence of the plexin-B1/Rac interaction is to modulate Sema4D ligand binding. This effectively classifies plexin-B1 as a downstream effector of Rac and is the first example of a small GTPase that directly regulates receptor function. An enhancement in the quantity of receptor at the cell membrane and minor changes in affinity for ligand contribute 
Vikis et al.

A
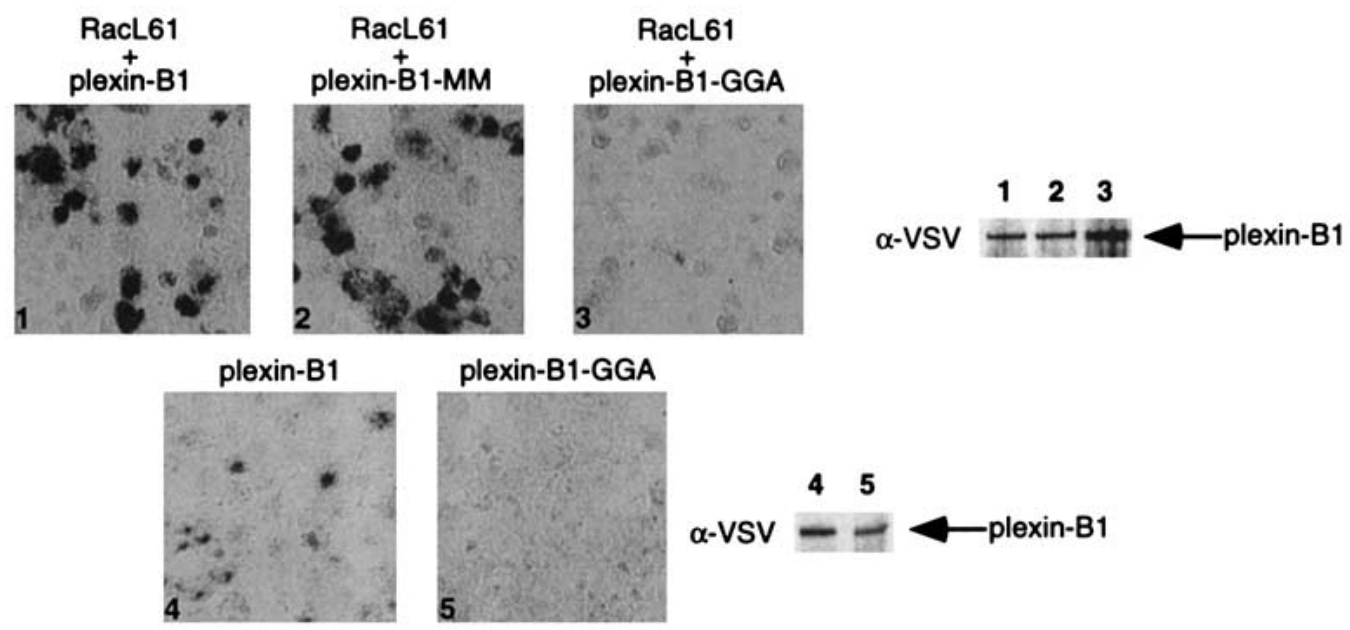

B
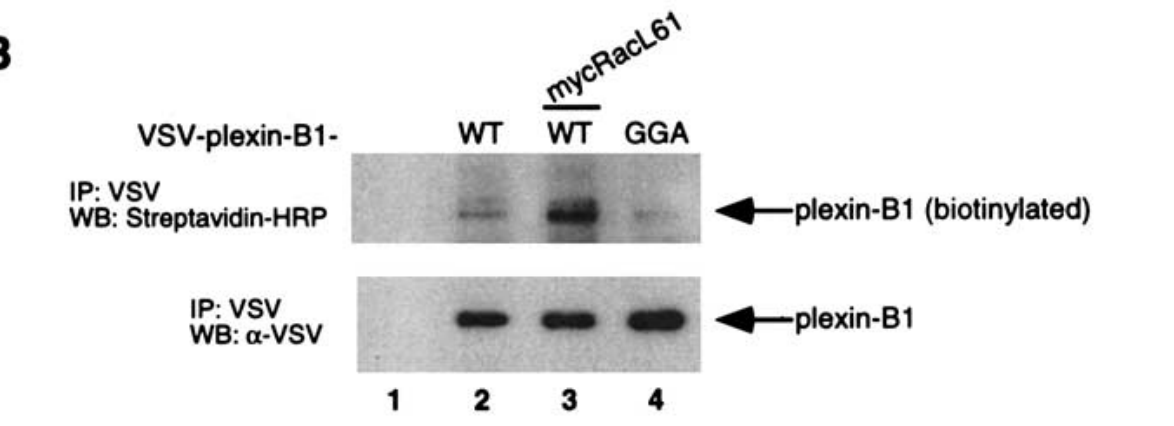

C
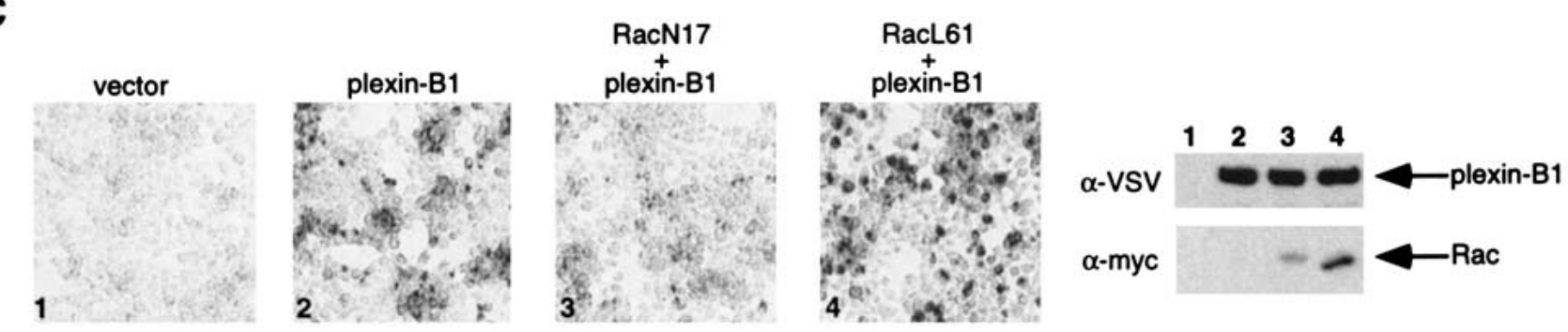

D
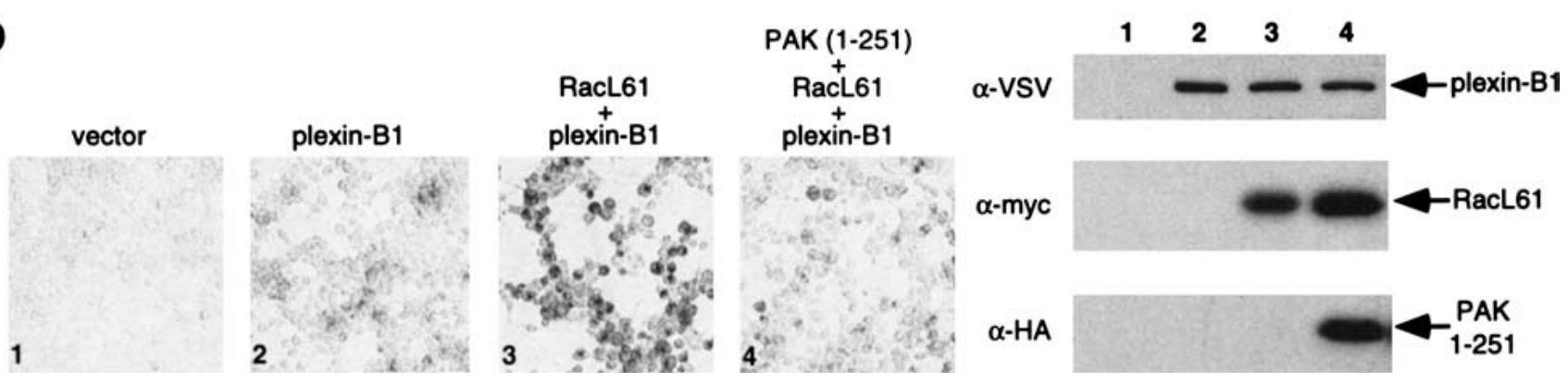

Figure 4. (A) The Rac binding deficient mutant plexin-B1-GGA is deficient in Sema4D binding and cannot be stimulated by RacL61. COS-7 cells were transfected with the indicated expression plasmids and treated with Sema4D as described in Figure 2A. Plexin-B1GGA showed lower basal and RacL61-stimulated Sema-4D binding (panel 1 vs. 3 and 4 vs. 5). (B) Cell surface expression of plexinB1-GGA is diminished. HEK293 cells were transfected with the indicated expression plasmids. Cells were biotinylated with SulfoNHS-LC-Biotin as described in Materials and Methods. Plexin-B1 and plexin-B1-GGA were immunoprecipitated with $\alpha$-VSV and detected by Western blot with streptavidin-HRP and $\alpha$-VSV Western blots. $(C)$ RacN17 inhibits the Sema4D/plexin-B1 interaction. HEK293 cells were transfected as indicated and subject to the same Sema4D treatment and detection as described in Figure 2A. (D) The N-terminal domain of p21-activated kinase (PAK) inhibits RacL61-induced Sema4D/plexin-B1 binding. HEK 293 cells were transfected as indicated and subject to the same Sema4D treatment and detection as described in Figure 2A. 
A

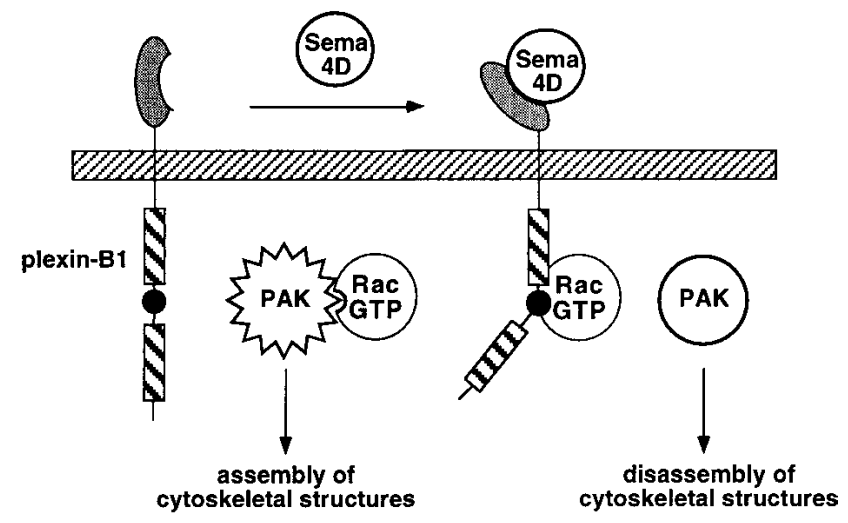

B

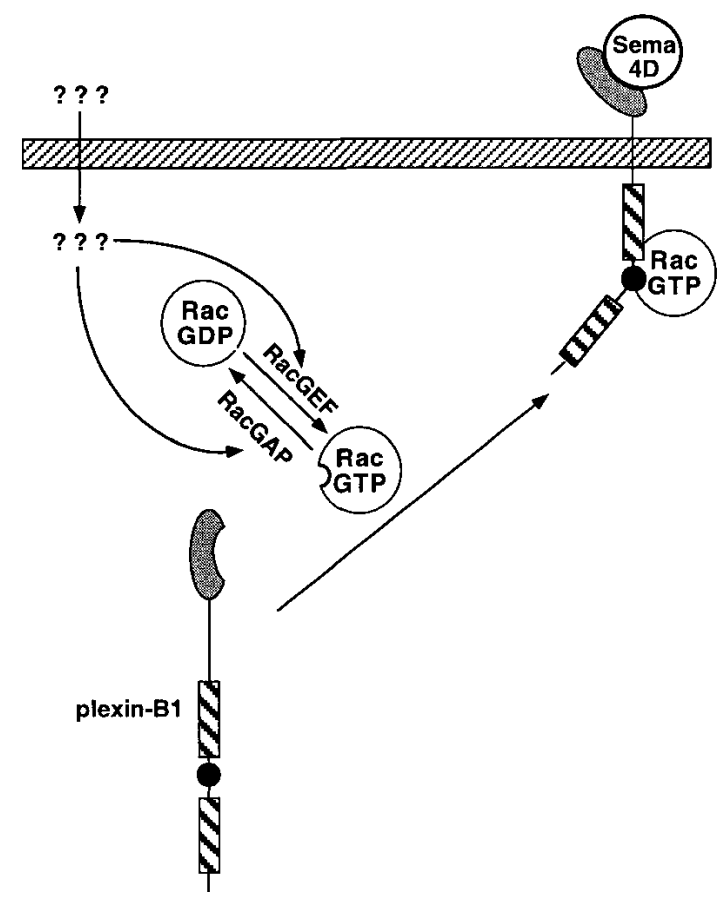

Figure 5. (A) Sema4D inhibits p21-activated kinase (PAK) activity and promotes growth cone collapse. Treatment of plexinB1 expressing neurons with Sema4D results in the recruitment and sequestration of active Rac. This inhibits the ability of Rac to activate PAK and thus results in the disassembly of cytoskeletal structures associated with growth cone collapse/turning. (B) Active Rac enhances the affinity of plexin-B1 for Sema4D and its localization at the cell surface. Expression of plexin-B1 at the cell surface requires endogenous GTP-Rac levels. Stimulation of Rac by signals acting on RacGEFs and/or RacGAPs result in enhanced localization of plexin-B1 at the membrane in addition to an enhanced affinity for Sema4D.

to this enhancement. Whether this is a result of enhanced recruitment to the cell surface and/or inhibition of receptor endocytosis is presently unclear. RhoA does not interact with plexin-B1 and does not stimulate Sema4D ligand binding, yet it has been reported to be activated by clustering of plexin-B1 receptor (Driessens et al. 2001). In Drosophila, plexB interacts with Rho and stimulates its activity (Hu et al. 2001). It appears that humans and flies use different mechanisms for plexin-B stimulation of Rho activity.

Our data also suggests that endogenous Rac-GTP is necessary for the maintenance of plexin-B1 at the cell surface. This is based on the observation that dominant negative Rac (RacN17), which inhibits endogenous Rac activation, effectively inhibited the plexin-B1/Sema4D interaction. Furthermore, the Rac binding defective mutant plexin-B1-GGA is compromised in the interaction with Sema4D. This lead us to postulate that factors that modulate Rac activation can enhance the sensitivity of the receptor/ligand interaction. It is possible that activation of a Rac-specific GEF and/or inactivation of a GAP may modulate the levels of plexin-B1 at the cell surface and its affinity for ligand (Fig. 5B). Hence, this suggests that engagement of plexin-B1 by Sema4D may be regulated by intracellular levels of Rac-GTP. What the biological consequence of this is is unknown, however this may be a mechanism by which Rac activation by one axon guidance cue can modulate the responsiveness of the axon growth cone to another guidance cue, such as Sema4D. It is worth noting that whether this model (Fig. 5) operates in axon growth cone guidance requires further analysis in neurons. Under physiological conditions the axon growth cone is exposed to multiple guidance cues. Therefore, Rac may act as a mediator for cross-talk between different axon guidance cues. Furthermore, our data suggest that signaling between plexin-B1 and Rac is bidirectional. Ligand-gated plexin-B1 can sequester Rac from activating other downstream effectors whereas active Rac can enhance the activity of plexin-B1.

\section{Materials and methods}

Plasmids

Cloning and bacterial expression of maltose binding protein (MBP)-plexin-B1, GST-RacN17/L61, and GST-PAK-RBD have been described previously (Vikis et al. 2000). pcDNA3plexB $1_{\text {cyto }}$ was constructed by PCR amplification of the cytoplasmic domain of plexin-B1 (amino acids 1512-2135). pcDNA3-HA-PAK was subcloned from pJ3H-hPAK1 (J. Chernoff, Fox Chase Cancer Center, Philadelphia, PA) and pcDNA3HA-PAK-1-251 was constructed by PCR amplification. The mammalian expression constructs pRK5-mycRhoL63, were kind gifts from A. Hall (University College London, UK). pRK5myc-RacN17/L61 was subcloned from pZIP-RacN17/L61 provided by C. Der (University of North Carolina, Chapel Hill). pcDNA3-VSV-plexin-B1, -VSV-plexin-A1 and-Flag-NP-1 were kind gifts from Z. He and P.M. Comoglio (Tamagnone et al. 1999). VSV-plexin-B1-GGA (L1849G,V1850G, and P1851A) and VSV-plexin-B1-MM (R1677M, R1678M) were constructed by PCR mutagenesis. Sema4D-SEAP and Sema3A-SEAP were gifts from Z. He (Harvard Medical School, Boston, MA) and A. Puschel (Max-Planck-Institute for Brain Research, Frankfurt, Germany), respectively.

\section{Cell culture and transfections}

HEK293, COS-7, and COS-1 cells were maintained in DMEM (GIBCO) supplemented with $10 \%$ fetal bovine serum (FBS). 
Transfections were performed using Lipofectamine (Invitrogen) as recommended by the manufacturer.

\section{In vitro binding}

In vitro binding experiments were performed as described previously (Vikis et al. 2000). Briefly, $10 \mu \mathrm{g}$ of MBP-B1 pre-bound to amylose beads were incubated in buffer containing $5 \mu \mathrm{g}$ of either GST-RacN17 or GST-RacL61/L61G37/L61C40 in the presence of increasing amounts of GST-PAK-RBD.

\section{PAK activity assay}

HEK293 cells were transfected with different combinations of $200 \mathrm{ng}$ of HA-PAK, $500 \mathrm{ng}$ plexin-B1 $1_{\text {cyto }}$ and $25 \mathrm{ng}$ or $50 \mathrm{ng}$ myc-RacL61. Forty-eight hours posttransfection cells were lysed in RIPA buffer $(0.1 \%$ SDS, $1 \%$ Triton X-100, $0.5 \%$ deoxycholate, $50 \mathrm{mM}$ Tris-Cl at $\mathrm{pH} 7.5,150 \mathrm{mM} \mathrm{NaCl}, 50 \mathrm{mM} \mathrm{NaF}$, $1 \mathrm{mM}$ EDTA, $1 \mathrm{mM}$ EGTA, $0.1 \mathrm{mM}$ phenylmethylsulfonyl fluoride, $5 \mu \mathrm{g} / \mathrm{mL}$ aprotinin, $5 \mu \mathrm{g} / \mathrm{mL}$ leupeptin, and $1 \mathrm{mM}$ sodium vanadate) and HA-PAK was immunoprecipitated with $\alpha-\mathrm{HA}$ (BAbCO). The kinase assay was carried out in $50 \mathrm{mM}$ HEPES (pH 7.4), $10 \mathrm{mM} \mathrm{MgCl} 2,2 \mathrm{mM} \mathrm{MnCl}_{2}, 0.2 \mathrm{mM}$ DTT, $0.2 \mathrm{mM}$ ATP, $10 \mu \mathrm{Ci}\left[\gamma_{-}{ }^{32} \mathrm{P}\right] \mathrm{ATP}$ and $10 \mu \mathrm{g}$ of histone H2B. Purified MBP-B $1_{\text {cyto }}$ protein was used to immunize rabbits for $\alpha$-plexinB1 antibody production (Pocono Rabbit Farm \& Laboratory, Inc., Canadensis, PA). Myc-RacL61 was detected with $\alpha$-myc9E10 (Covance). Phosphorylated PAK was detected by anti-phospho PAK (J. Chernoff, Fox Chase Cancer Center, Philadelphia, PA).

\section{Semaphorin staining assays}

COS-7, COS-1, and HEK293 cells were transfected in multi-well plates in duplicate with $1 \mu \mathrm{g}$ of VSV-plexin-B1 and $0.1 \mu \mathrm{g}$ of myc-RacL61 expression constructs. Forty-eight hours posttransfection, cells were treated with 0.5-5 nM AP-Sema4D for 1 $\mathrm{h}$ in serum-free DMEM media (GIBCO BRL). Cells were washed three times with buffer (Hanks' Balanced Salt Solution, GIBCO BRL), $20 \mathrm{mM}$ HEPES at $\mathrm{pH} 7,0.5 \mathrm{mg} / \mathrm{mL}$ BSA) and fixed with $4 \%$ paraformaldehyde, $10 \%$ sucrose in PBS for $5 \mathrm{~min}$. Cells were placed at $65^{\circ} \mathrm{C}$ for $1 \mathrm{~h}$ to inactivate endogenous phosphatases. Cells were developed in TMNT buffer (100 mM Tris-Cl at $\mathrm{pH}$ 9.5, $100 \mathrm{mM} \mathrm{NaCl}, 50 \mathrm{mM} \mathrm{MgCl}, 0.1 \%$ Tween-20) with 0.17 $\mathrm{mg} / \mathrm{mL}$ 5-bromo-4-chloro-3-indolyl phosphate (BCIP) and 0.33 $\mathrm{mg} / \mathrm{mL}$ nitrotetrazolium blue (NBT). Photos of stained cells were taken with a Leica DM IRB light microscope over the course of $1-3 \mathrm{~d}$ for COS-7 cells and $2-12 \mathrm{~h}$ for 293 cells. A duplicate plate of cells was lysed in RIPA buffer and plexin-B1 was detected by $\alpha$-VSV (Boehringer Mannheim) Western blot.

Alkaline phosphatase semaphorin fusion proteins were expressed by transfection of HEK293 cells with Sema4D-SEAP or Sema3A-SEAP expression constructs. Cells were grown in $1 \%$ FBS/DMEM and media were collected and concentrated prior to determination of concentration by pNPP activity (Flanagan and Leder 1990).

\section{Sema4D binding assays}

COS-7 and HEK293 cells were transfected in triplicate similar to the staining assays. Forty-eight hours posttransfection cells were incubated with varying concentrations of Sema4D for $1 \mathrm{~h}$. Cells were washed with buffer (Hanks' Balanced Salt Solution, $20 \mathrm{mM}$ HEPES at $\mathrm{pH} 7,0.5 \mathrm{mg} / \mathrm{mL}$ BSA) and then lysed in 10 $\mathrm{mM}$ Tris- $\mathrm{Cl}(\mathrm{pH} 8), 1 \%$ TX-100. Cell lysates were then cleared at $15,000 \mathrm{~g}$ and heated to $65^{\circ} \mathrm{C}$ for $20 \mathrm{~min}$. Alkaline phosphatase activity was measured by para-nitrophenyl phosphate (pNPP) hydrolysis in a reaction buffer containing $10 \%$ diethanolamine (pH 9.8), $0.5 \mathrm{mM} \mathrm{MgCl}_{2}$, and $10 \mathrm{mM}$ pNPP. Absorbance at 405 $\mathrm{nM}$ was measured. Absorbance values from vector-transfected cells were subtracted.

\section{Proteinase K treatment}

HEK293 cells were transfected with $1 \mu \mathrm{g}$ of VSV-plexin-B1 and $0.1 \mu \mathrm{g}$ of myc-RacL61 expression constructs. Forty-eight hours posttransfection cells were washed in PBS and incubated in PBS with $0.2 \mathrm{mg} / \mathrm{mL}$ proteinase $\mathrm{K}$ for $1 \mathrm{~h}$ at $37^{\circ} \mathrm{C}$. Cells were then washed with PBS (plus $1 \mathrm{mM}$ PMSF), lysed in NP-40 buffer (20 $\mathrm{mM}$ Tris-Cl at $\mathrm{pH} 7.5,100 \mathrm{mM} \mathrm{NaCl}, 1 \% \mathrm{NP}-40$ ) and subject to SDS-PAGE and Western blot with $\alpha$-B1 antibody.

\section{Cell surface biotinylation}

HEK293 cells were transfected as indicated. Forty-eight hours posttransfection, cells were washed with PBS (pH 8) and incubated with $0.5 \mathrm{mg} / \mathrm{mL}$ Sulfo-NHS-LC-Biotin (Pierce) in PBS (pH 8) for $40 \mathrm{~min}$ at room temperature. Cells were washed in PBS (pH 8), $50 \mathrm{mM}$ Tris-Cl (pH 8). Cells were then lysed in NP-40 buffer plus $0.1 \%$ TX-100 and subject to immunoprecipitation with $\alpha$-VSV. Samples were subject to SDS-PAGE and Western blot with $\alpha$-VSV and streptavidin-HRP (Amersham).

\section{Acknowledgments}

We wish to thank Drs. Z. He, J. Chernoff, A. Hall, C. Der, M. Tessier-Lavigne, A. Chedotal, P.M. Comoglio, and L. Tamagnone for reagents and J. Aurandt and J.E. Dixon for helpful comments. This work was supported by grants from National Institutes of Health and Walther Cancer Institute (K.L.G.). K.L.G. is a MacArthur Fellow.

The publication costs of this article were defrayed in part by payment of page charges. This article must therefore be hereby marked "advertisement" in accordance with 18 USC section 1734 solely to indicate this fact.

\section{References}

Bentley, D. and O'Connor, T.P. 1994. Cytoskeletal events in growth cone steering. Curr. Opin. Neurobiol. 4: 43-48.

Buchwald, G., Hostinova, E., Rudolph, M.G., Kraemer, A., Sickmann, A., Meyer, H.E., Scheffzek, K., and Wittinghofer, A. 2001. Conformational switch and role of phosphorylation in PAK activation. Mol. Cell. Biol. 21: 5179-5189.

Culotti, J.G. and Kolodkin, A.L. 1996. Functions of netrins and semaphorins in axon guidance. Curr. Opin. Neurobiol. 6: $81-88$.

Dharmawardhane, S., Sanders, L.C., Martin, S.S., Daniels, R.H., and Bokoch, G.M. 1997. Localization of p21-activated kinase 1 (PAK1) to pinocytic vesicles and cortical actin structures in stimulated cells. J. Cell. Biol. 138: 1265-1278.

Driessens, M.H., Hu, H., Nobes, C.D., Self, A., Jordens, I., Goodman, C.S., and Hall, A. 2001. Plexin-B semaphorin receptors interact directly with active Rac and regulate the actin cytoskeleton by activating Rho. Curr. Biol. 11: 339-344.

Fan, J., Mansfield, S.G., Redmond, T., Gordon-Weeks, P.R., and Raper, J.A. 1993. The organization of F-actin and microtubules in growth cones exposed to a brain-derived collapsing factor. J. Cell. Biol. 121: 867-878.

Flanagan, J.G. and Leder, P. 1990. The kit ligand: A cell surface molecule altered in steel mutant fibroblasts. Cell 63: 185194. 
Frost, J.A., Khokhlatchev, A., Stippec, S., White, M.A., and Cobb, M.H. 1998. Differential effects of PAK1-activating mutations reveal activity-dependent and -independent effects on cytoskeletal regulation. J. Biol. Chem. 273: 2819128198.

Hing, H., Xiao, J., Harden, N., Lim, L., and Zipursky, S.L. 1999. Pak functions downstream of Dock to regulate photoreceptor axon guidance in Drosophila. Cell 97: 853-863.

Hu, H., Marton, T.F., and Goodman, C.S. 2001. Plexin b mediates axon guidance in Drosophila by simultaneously inhibiting active rac and enhancing rhoa signaling. Neuron 32: $39-51$.

Jin, Z. and Strittmatter, S.M. 1997. Rac1 mediates collapsin-1induced growth cone collapse. J. Neurosci. 17: 6256-6263.

Knaus, U.G. and Bokoch, G.M. 1998. The p21Rac/Cde42-activated kinases (PAKs). Int. J. Biochem. Cell. Biol. 30: 857862

Kuhn, T.B., Brown, M.D., Wilcox, C.L., Raper, J.A., and Bamburg, J.R. 1999. Myelin and collapsin-1 induce motor neuron growth cone collapse through different pathways: Inhibition of collapse by opposing mutants of rac1. I. Neurosci. 19: $1965-1975$.

Lamarche, N., Tapon, N., Stowers, L., Burbelo, P.D., Aspenstrom, P., Bridges, T., Chant, J., and Hall, A. 1996. Rac and Cdc42 induce actin polymerization and $\mathrm{G}_{1}$ cell cycle progression independently of p65PAK and the JNK/SAPK MAP kinase cascade. Cell 87: 519-529.

Lim, L., Manser, E., Leung, T., and Hall, C. 1996. Regulation of phosphorylation pathways by 21 GTPases. The p21 Rasrelated Rho subfamily and its role in phosphorylation signalling pathways. Eur. J. Biochem. 242: 171-185.

Liu, B.P. and Strittmatter, S.M. 2001. Semaphorin-mediated axonal guidance via Rho-related G proteins. Curr. Opin. Cell. Biol. 13: 619-626.

Luo, Y., Raible, D., and Raper, J.A. 1993. Collapsin: A protein in brain that induces the collapse and paralysis of neuronal growth cones. Cell 75: 217-227.

Messersmith, E.K., Leonardo, E.D., Shatz, C.J., Tessier-Lavigne, M., Goodman, C.S., and Kolodkin, A.L. 1995. Semaphorin III can function as a selective chemorepellent to pattern sensory projections in the spinal cord. Neuron 14: 949-959.

Newsome, T.P., Schmidt, S., Dietzl, G., Keleman, K., Asling, B., Debant, A., and Dickson, B.J. 2000. Trio combines with dock to regulate Pak activity during photoreceptor axon pathfinding in Drosophila. Cell 101: 283-294.

O'Connor, T.P. and Bentley, D. 1993. Accumulation of actin in subsets of pioneer growth cone filopodia in response to neural and epithelial guidance cues in situ. J. Cell. Biol. 123: 935-948.

Puschel, A.W. 1996. The semaphorins: A family of axonal guidance molecules? Eur. J. Neurosci. 8: 1317-1321.

Puschel, A.W., Adams, R.H., and Betz, H. 1995. Murine semaphorin $\mathrm{D} /$ collapsin is a member of a diverse gene family and creates domains inhibitory for axonal extension. Neuron 14: 941-948.

Rohm, B., Ottemeyer, A., Lohrum, M., and Puschel, A.W. 2000a. Plexin/neuropilin complexes mediate repulsion by the axonal guidance signal semaphorin 3A. Mech. Dev. 93: 95-104.

Rohm, B., Rahim, B., Kleiber, B., Hovatta, I., and Puschel, A.W. $2000 \mathrm{~b}$. The semaphorin $3 \mathrm{~A}$ receptor may directly regulate the activity of small GTPases. FEBS Lett. 486: 68-72.

Sells, M.A., Knaus, U.G., Bagrodia, S., Ambrose, D.M., Bokoch, G.M., and Chernoff, J. 1997. Human p21-activated kinase (Pak1) regulates actin organization in mammalian cells. Curr. Biol. 7: 202-210.
Shamah, S.M., Lin, M.Z., Goldberg, J.L., Estrach, S., Sahin, M., Hu, L., Bazalakova, M., Neve, R.L., Corfas, G., Debant, A., et al. 2001. EphA receptors regulate growth cone dynamics through the novel guanine nucleotide exchange factor ephexin. Cell 105: 233-244.

Takahashi, T., Fournier, A., Nakamura, F., Wang, L.H., Murakami, Y., Kalb, R.G., Fujisawa, H., and Strittmatter, S.M. 1999. Plexin-neuropilin-1 complexes form functional semaphorin-3A receptors. Cell 99: 59-69.

Tamagnone, L., Artigiani, S., Chen, H., He, Z., Ming, G.I., Song, H., Chedotal, A., Winberg, M.L., Goodman, C.S., Poo, M., et al. 1999. Plexins are a large family of receptors for transmembrane, secreted, and GPI-anchored semaphorins in vertebrates. Cell 99: 71-80.

Tessier-Lavigne, M. and Goodman, C.S. 1996. The molecular biology of axon guidance. Science 274: 1123-1133.

Vikis, H.G., Li, W., He, Z., and Guan, K.L. 2000. The semaphorin receptor plexin-B1 specifically interacts with active Rac in a ligand-dependent manner. Proc. Natl. Acad. Sci. 97: $12457-12462$.

Wong, K., Ren, X.R., Huang, Y.Z., Xie, Y., Liu, G., Saito, H., Tang, H., Wen, L., Brady-Kalnay, S.M., Mei, L., et al. 2001. Signal transduction in neuronal migration. Roles of gtpase activating proteins and the small gtpase cdc42 in the slitrobo pathway. Cell 107: 209-221. 


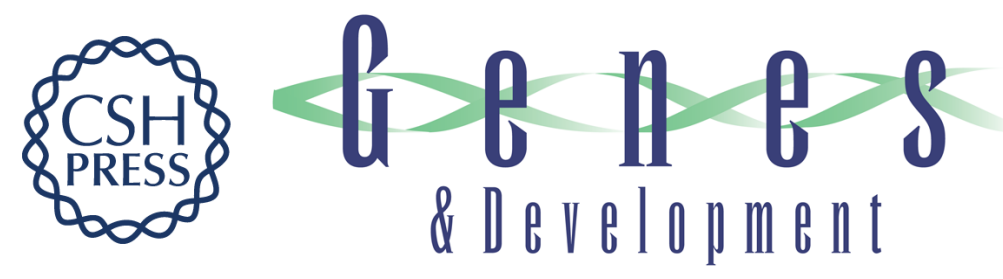

\section{The Plexin-B1/Rac interaction inhibits PAK activation and enhances Sema4D ligand binding}

Haris G. Vikis, Weiquan Li and Kun-Liang Guan

Genes Dev. 2002, 16:

Access the most recent version at doi:10.1101/gad.966402

References This article cites 30 articles, 9 of which can be accessed free at: http://genesdev.cshlp.org/content/16/7/836.full.htmI\#ref-list-1

License

Email Alerting

Receive free email alerts when new articles cite this article - sign up in the box at the top Service right corner of the article or click here.

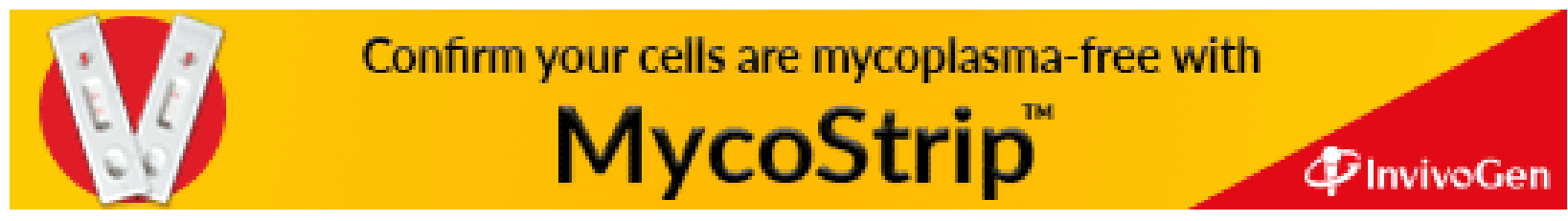

